Artigo / Article

\title{
Doadores de sangue de primeira vez e comportamento de retorno no hemocentro público do Recife
}

\section{First time blood donors and the pattern of return visits to the public blood bank of Recife}

Fábia Michelle Rodrigues de Araújol

Katia Virginia de Oliveira Feliciano ${ }^{2}$

Marina Ferreira de Medeiros Mendes ${ }^{3}$

José Natal Figueiroa ${ }^{2}$

\begin{abstract}
Objetivo: Analisar o perfil dos doadores de sangue conforme o periodo da primeira doação e comportamento de retorno no hemocentro público do Recife. Métodos: Estudo retrospectivo com 363 doadores, no qual se utilizou o banco de dados do Sistema de Banco de Sangue (SBS) referente ao periodo de 01 de janeiro de 1998 a 13 de junho de 2008. Na análise avaliaram-se a mediana do número de doações por sexo, idade e escolaridade, além da associação entre o período da primeira doação e o sexo, idade à primeira doação e fator Rh. Para 261 (excluídos 100 doadores de primeira vez e dois por imprecisão da data do retorno), o comportamento de retorno. Resultados: Aproximadamente 77,1\% eram homens, 40,5\% entre $25-34$ anos de idade, 47,7\% haviam completado o segundo grau e 54\% doaram espontaneamente. A mediana do número de doações foi maior entre os homens $(p=0,000)$ mais velhos $(p=0,000)$. A participação feminina cresceu no periodo atual $(30,6 \%)$ em comparação a 1998-2000 (4,8\%), mas o percentual superior dos doadores de primeira vez era referente a homem ( $p=0,003)$. Daqueles que doaram cinco ou mais vezes, 43,8\% retornaram para próxima doação com intervalo menor que seis meses. Prevaleceu o sexo masculino entre doadores de repetição $(p=0,011)$. Conclusões: A grande maioria dos doadores de repetição (intervalo de tempo entre as duas últimas doações igual ou inferior a 13 meses) ainda não está próximo do número máximo de doação anual. É fundamental concentrar a atenção neste grupo potencialmente mais receptivo para aumentar efetividade do recrutamento.
\end{abstract}

Descritores: Doadores de sangue; Bancos de sangue

\section{Introdução}

Os avanços tecnológicos, que possibilitam a realização de procedimentos terapêuticos de alta complexidade, associados às mudanças no perfil epidemiológico da população, com destaque para o crescimento da violência, aumentam a necessidade de sangue e produtos hemoterá- picos. Segundo a Organização Mundial de Saúde (OMS), o número de doações de sangue adequado para um país estaria entre 3\% e 5\% da população, quantitativo já alcançado nos países europeus. Em 1998, o Ministério da Saúde definiu as diretrizes e metas para a hemoterapia brasileira, dentre as quais: coletar um número de bolsas de sangue correspondentes a $3 \%$ da população, sendo $80 \%$ oriundas de

Fundação de Hematologia e Hemoterapia de Pernambuco (Hemope) - Recife (PE), Brasil.

${ }^{I}$ Instituto de Medicina Integral Professor Fernando Figueira - Recife (PE), Brasil.

${ }_{2}^{2}$ Diretoria de Ensino da Pós-graduação Stricto Sensu em Saúde Materno Infantil do Instituto de Medicina Integral Professor Fernando Figueira - Recife (PE), Brasil.

${ }^{3}$ Grupo de Estudos em Avaliação de Saúde da Diretoria de Pesquisa do Instituto de Medicina Integral Professor Fernando Figueira - Recife (PE), Brasil.

Conflito de interesse: sem conflito de interesse

Correspondência: Fábia Michelle Rodrigues de Araújo

Rua do Futuro, 1150/ apto 206 - Jaqueira

52050-010 - Recife (PE), Brasil

Tel.: (55 81) 99529355

E-mail:fabiamra@yahoo.com.br 
doações espontâneas e obter $60 \%$ de doações de repetição. ${ }^{(1)}$ Todavia, observa-se tendência estacionária das doações que representam $1,70 \%$ da população. ${ }^{(2)}$

Em um contexto no qual a exigência de crescimento das doações convive com os obstáculos na captação e no incremento do número de doadores regulares, a busca por maior qualidade dos produtos sanguíneos compromete ainda mais a disponibilidade de sangue. ${ }^{(3)}$ Embora seja essencial empreender esforços na captação de pessoas sem antecedentes de doação, uma maior frequência de doações de retorno possibilitaria, ao mesmo tempo, a ampliação do atendimento à demanda crescente por sangue, a garantia de bolsas de sangue com maior segurança para o receptor e a redução do custo da coleta: os doadores regulares apresentam consistentemente uma menor inaptidão sorológica, diminuindo o número de exames desprezados nos retestes. ${ }^{(4,5)}$

Autores internacionais mostram que a análise da frequência de doação pode ser usada como preditora da probabilidade de que o doador venha a se tornar de repetição em longo prazo. Nesse sentido, o intervalo de tempo entre a primeira e a segunda doação está associado ao número total de doações ${ }^{(6-10)}$ Constata-se a forte associação entre o gênero, a idade, a escolaridade e o tipo de doador. Essas pesquisas são fundamentais para o ajustamento das estratégias voltadas ao recrutamento e ao estímulo da regularidade das doações, logo, para a manutenção de um estoque de sangue estável, seguro e adequado. ${ }^{(5,8,10,11)}$ Daí a importância deste artigo, que tem como objetivo analisar o perfil dos doadores de sangue segundo o período da primeira doação e o comportamento de retorno no hemocentro público da cidade do Recife.

\section{Métodos}

Estudo descritivo, retrospectivo, no qual se utilizou o banco de dados do Sistema de Banco de Sangue (SBS), referente aos prontuários dos doadores de sangue do Hemocentro Recife da Fundação de Hematologia e Hemoterapia de Pernambuco (Hemope), no período de 01 de janeiro de 1997 a 13 de junho de 2008. Estes doadores representam subamostra selecionada a partir de estudo transversal, efetuado entre 21 de janeiro e 30 de maio de 2008 , para conhecer a percepção do atendimento na doação de sangue neste hemocentro, que representa o único serviço de hemoterapia público de apoio à rede de serviços de saúde do Recife e Região Metropolitana. O Comitê de Ética em Pesquisa do Hemope aprovou o projeto em 31/10/2007.

Para cálculo do tamanho da amostra no desenho transversal estimaram-se $10 \%$ de avaliações negativas da doação de sangue, conforme pesquisa realizada no hemocentro em $2007,{ }^{(12)}$ nível de confiança de $95 \%$ e erro de $2,5 \%$. Com base nessas informações, a amostra teria tamanho igual a 553 doadores. Na amostragem, em cada turno, utilizou-se listagem organizada sequencialmente, sendo a décima nona convidada a responder o questionário aplicado por umas das autoras, em uma sala do serviço, após finalizar procedimento. Ao encerrar o período de coleta, a amostra final constava de 527 doadores que assinaram o termo de consentimento livre e esclarecido. Cobriram-se 43 turnos da manhã e 37 da tarde para se obter amostra representativa. Houve 44 recusas, duas exclusões (problema cognitivo visível e dispensa da bolsa por inadequação clínica) e 13 perdas (aceitaram participar, mas não foram localizados após doação).

O estudo retrospectivo contemplou 363 doadores, tendo sido excluídos 164 com histórico de doação no Hemocentro Recife anterior ao SBS, o qual foi implantado no território nacional em dezembro de 1997, pois, para estes, a única informação disponível era o número total de doações que cada doador realizara antes da implantação do Sistema. Convém ressaltar que esta subamostra, afora um percentual maior de solteiros, apresentou perfil sociodemográfico, experiência de familiares com doação, caráter espontâneo e motivos para doação atual semelhantes à amostra do corte transversal. Para a coleta de dados secundários foi utilizado formulário próprio no qual se registraram o tipo sanguíneo e as datas das doações realizadas nesta unidade hemoterápica que constavam do SBS.

A análise obedeceu às seguintes etapas: (1) para 363 que compunham a subamostra, o número de doações foi associado ao sexo, idade e escolaridade, sendo avaliada também a associação entre período da primeira doação e sexo, idade à primeira doação e fator Rh; (2) Dos 363 foram excluídos os 100 doadores de primeira vez que não haviam retornado para outra doação de sangue até finalizar o levantamento dos dados secundários e dois por imprecisão da data do retorno; restando 263, estudou-se a associação entre o intervalo do primeiro retorno, número total de doações, sexo, idade à primeira doação, fator Rh e tipo de doador; (3) para os 155 doadores de repetição, identificados na subamostra, o intervalo de tempo entre a primeira e a última doação foi analisado por meio da curva de sobrevivência.

Adotou-se a definição da Resolução Nº149/2001, do Sistema Nacional de Informação da Produção Hemoterápica ${ }^{(13)}$ quanto ao tipo de doador, classificando-se como de repetição aquele doador cujo intervalo de tempo entre as duas últimas doações no Hemocentro Recife foi igual ou inferior a 13 meses, e como esporádico aquele que doou eventualmente num intervalo superior a 13 meses. Além disso, definiu-se como doador que já foi de repetição aquele doador que, em algum momento de sua história de doação no referido serviço, esteve no estado de doador de repetição.

A existência de diferenças significativas foi avaliada, com um nível de confiança de 95\%, entre: (a) as medianas com teste de Kruskal-Wallis; e (b) as proporções com o quiquadrado. Montou-se uma coorte retrospectiva de doadores de repetição disponíveis seguidos até a finalização da coleta de dados secundários, realizando análise do tempo até o 
último retorno pelo método de Kaplan Meier. ${ }^{(14)} \mathrm{A}$ análise estatística foi realizada com software Stata 9.2.

\section{Resultados}

Dos 363 doadores, 77,1\% eram do sexo masculino, $53,7 \%$ residiam na cidade do Recife e $40,5 \%$ tinham entre 25 e 34 anos, com medianas de idades diferentes $(\mathrm{p}=0,023)$ para homens (28 anos) e mulheres (31 anos). Acerca da educação formal, $47,7 \%$ haviam completado o segundo grau, $7,7 \%$ concluíram curso superior e $0,8 \%$ nunca estudaram. Perto de $48,0 \%$ eram casados ou tinham uma relação estável e 71,4\% integravam a população economicamente ativa. Os familiares de $62,3 \%$ já doaram sangue e de quase duas quintas partes receberam transfusão sanguínea. Para 54,0\%, a doação foi espontânea, sendo de convocação em 3,6\% dos casos $(69,2 \%$ com fator Rh negativo). Os principais motivos da doação atual foram solicitação de amigos e familiares $(41,0 \%)$, tempo de retorno $(33,3 \%)$ e solidariedade $(12,1 \%)$. (Tabela 1).

O número de doações evidenciou-se estatisticamente associada ao sexo e à idade $(\mathrm{p}<0,001)$. A mediana do número de doações para o sexo masculino foi igual a três doações

\begin{tabular}{lcc} 
Tabela 1. Características sociodemográficas, antecedentes de doação/ \\
transfusão entre familiares e amigos, tipo e motivos da doação atual dos \\
doadores de sangue entrevistados. Hemocentro do Recife, Brasil, 2008 \\
\hline Características dos doadores & $\mathrm{n}$ & $(\%)$ \\
\hline Sexo & & \\
Masculino & 280 & $(77,1)$ \\
Feminino & 83 & $(22,9)$ \\
Idade (anos) & & \\
18-24 & 108 & $(29,7)$ \\
$25-34$ & 147 & $(40,5)$ \\
35-44 & 68 & $(18,7)$ \\
$\geq 45$ & 40 & $(11,1)$ \\
Escolaridade (anos) & 3 & $(1,9)$ \\
Nenhum & 19 & $(5,2)$ \\
1-4 & 70 & $(19,3)$ \\
5-8 & 206 & $(56,7)$ \\
9-11 & 65 & $(17,9)$ \\
$\geq 12$ & & \\
Reside na cidade do Recife & 195 & $(53,7)$ \\
Casado/vive junto & 174 & $(48,0)$ \\
Inserido no mercado de trabalho & 259 & $(71,4)$ \\
Familiares já doaram sangue & 226 & $(62,3)$ \\
Familiares submeteram-se à transfusão de sangue & 144 & $(39,8)$ \\
Doação de sangue atual & & \\
Espontânea & 196 & $(54,0)$ \\
Reposição & 154 & $(42,4)$ \\
Convocação & 13 & $(3,6)$ \\
O que motivou doação atual & & \\
Amigos/familiares & 149 & $(41,0)$ \\
É tempo de retornar & 121 & $(33,3)$ \\
Solidariedade & 44 & $(12,1)$ \\
Campanha & 27 & $(7,4)$ \\
Outros & 22 & $(6,2)$ \\
\hline
\end{tabular}

(1-19 doações). Já o sexo feminino teve mediana igual a duas doações (1-7 doações). A frequência de doações foi maior com a idade, sendo aqueles entre 45 e 54 anos os que apresentaram maiores valores: mediana de 4,5 doações (1-19 doações). Observou-se um crescimento de doação com a escolaridade, mas sem significância estatística; os doadores que tinham entre 9 e 11 anos de educação formal, com mediana igual a três doações, tiveram a maior amplitude (uma a 19 doações).

Quando se considerou a primeira doação (Tabela 2), pôde-se observar que uma proporção significativamente maior entre os doadores que fizeram a primeira doação no período de 2007-2008, em comparação com aqueles que doaram entre 1998-2000, pertenciam ao sexo masculino $(p=0,003)$. No período estudado, a frequência de doadores com idade entre 18 e 34 anos na primeira doação manteve-se ao redor de $78,2 \%$, com a distribuição etária concentrada entre 18 e 24 anos (48,4\%). Entre 2001 e 2003 ocorreu uma diminuição na proporção de doadores de primeira vez cujo fator Rh era negativo, o qual permaneceu até o período atual, mas sem significância estatística.

É possível observar (Tabela 3) que ao redor de uma terça parte dos doadores com, no mínimo, duas doações realizou o seu primeiro retorno em um período igual ou menor do que seis meses. Para uma proporção significativamente maior $(\mathrm{p}=0,011)$ daqueles que doaram cinco ou mais vezes, $\mathrm{o}$ primeiro retorno ocorreu em até seis meses $(43,8 \%)$ e apenas $32,7 \%$ deste grupo não haviam retornado até 12 meses. Dentre aqueles que doaram duas vezes, verificou-se um elevado retorno em períodos iguais ou maiores do que 19 meses $(47,3 \%)$. O sexo não afetou significativamente o tempo do primeiro retorno, embora mais homens $(35,2 \%)$, em comparação com as mulheres $(25,5 \%)$, tenham retornado em até seis meses.

O tempo do primeiro retorno (Tabela 3) também não estava associado com a idade à primeira doação, tampouco com o fator $\mathrm{Rh}$, embora um percentual maior de doadores com fator $\mathrm{Rh}$ negativo tenha retornado em até seis meses. O tipo de doador foi significativamente diferente em relação ao período de tempo do primeiro retorno $(p=0,000)$. Para $43,9 \%$ dos doadores de repetição, o primeiro retorno ocorreu em até seis meses, com apenas $28,4 \%$ deste grupo não retornando até 12 meses. Todos os doadores esporádicos retornaram após 12 meses. Aqueles que já haviam sido de repetição em algum momento da sua trajetória como doador fizeram o primeiro retorno em um tempo semelhante aos doadores de repetição.

Um percentual significativamente maior $(\mathrm{p}=0,011)$ dos homens $(63,3 \%)$ quando comparado com as mulheres $(43,1 \%)$ era doador de repetição. A doação esporádica foi duas vezes mais frequente entre as mulheres $(35,3 \%)$ do que para os homens (17,6\%). Ambos os sexos apresentaram comportamento semelhante no que diz respeito a ter sido, em algum momento, doador de repetição. Não foi observada associação entre o tipo de doador e o fator Rh. 
Tabela 2. Sexo, idade a primeira doação e fator $\mathrm{Rh}$, segundo o período da primeira doação de sangue. Hemocentro Recife, Brasil, 2008

\begin{tabular}{|c|c|c|c|c|c|c|c|c|c|}
\hline \multirow{3}{*}{$\begin{array}{l}\text { Variáveis } \\
(\mathrm{n}=363)\end{array}$} & \multicolumn{8}{|c|}{ Período da primeira doação (anos) } & \multirow{3}{*}{$\begin{array}{c}\text { Valor de } \\
\qquad p\end{array}$} \\
\hline & \multicolumn{2}{|c|}{$\begin{array}{c}1998-2000 \\
(N=42)\end{array}$} & \multicolumn{2}{|c|}{$\begin{array}{c}2001-2003 \\
(\mathrm{~N}=82)\end{array}$} & \multicolumn{2}{|c|}{$\begin{array}{c}2004-2006 \\
(\mathrm{~N}=92)\end{array}$} & \multicolumn{2}{|c|}{$\begin{array}{c}2007-2008(*) \\
(\mathrm{N}=147)\end{array}$} & \\
\hline & $\mathrm{n}^{\circ}$ & $\%$ & $\mathrm{n}^{\circ}$ & $\%$ & $\mathrm{n}^{\circ}$ & $\%$ & $\mathbf{n}^{\circ}$ & $\%$ & \\
\hline \multicolumn{10}{|l|}{ Sexo } \\
\hline Masculino & 40 & 95,2 & 67 & 81,7 & 71 & 77,2 & 102 & 69,4 & 0,003 \\
\hline Feminino & 2 & 4,8 & 15 & 18,3 & 21 & 22,8 & 45 & 30,6 & \\
\hline \multicolumn{10}{|l|}{ Idade (anos) } \\
\hline $18-24$ & 20 & 47,6 & 45 & 54,1 & 41 & 44,6 & 70 & 47,6 & 0,632 \\
\hline $25-34$ & 12 & 28,6 & 20 & 24,4 & 34 & 36,9 & 42 & 28,6 & \\
\hline $35-44$ & 7 & 16,7 & 10 & 12,2 & 14 & 15,2 & 25 & 17,0 & \\
\hline$\geq 45$ & 3 & 7,1 & 7 & 9,3 & 3 & 3,3 & 8 & 6,8 & \\
\hline \multicolumn{10}{|l|}{ Fator Rh } \\
\hline Positivo & 34 & 80,9 & 72 & 87,8 & 81 & 88,0 & 131 & 89,1 & 0,564 \\
\hline Negativo & 8 & 19,1 & 10 & 12,2 & 11 & 12,0 & 16 & 10,9 & \\
\hline
\end{tabular}

(*) até 13 de junho de 2008

Tabela 3. Intervalo do primeiro retorno, por número total de doações, sexo, idade a primeira doação, fator Rh e padrão de doação de doadores com no mínimo duas doações. Hemocentro do Recife, Brasil, 2008

\begin{tabular}{|c|c|c|c|c|}
\hline & \multicolumn{3}{|c|}{ Meses } & \\
\hline$(\mathrm{n}=261 *)$ & $\leq 6$ & $7-12$ & $13-18$ & Valor de \\
\hline
\end{tabular}

Número total de

doações

\begin{tabular}{|c|c|c|c|c|c|c|c|c|c|}
\hline 2 & 16 & 21,6 & 13 & 17,6 & 10 & 13,5 & 35 & 47,3 & 0,011 \\
\hline 3 & 9 & 24,3 & 12 & 32,5 & 6 & 16,2 & 10 & 27,0 & \\
\hline 4 & 10 & 32,3 & 7 & 22,5 & 3 & 9,7 & 11 & 35,5 & \\
\hline$\geq 5$ & 52 & 43,8 & 28 & 23,5 & 14 & 11,7 & 25 & 21,0 & \\
\hline \multicolumn{10}{|l|}{ Sexo } \\
\hline Masculino & 74 & 35,2 & 43 & 20,5 & 26 & 12,4 & 67 & 31,9 & \multirow[t]{2}{*}{0,218} \\
\hline Feminino & 13 & 25,5 & 17 & 33,3 & 7 & 13,6 & 14 & 27,6 & \\
\hline \multicolumn{10}{|l|}{ Idade (anos) } \\
\hline $18-24$ & 47 & 35,4 & 24 & 18,0 & 16 & 12,0 & 46 & 34,6 & \multirow[t]{4}{*}{0,436} \\
\hline $25-34$ & 23 & 29,5 & 20 & 25,6 & 11 & 14,1 & 24 & 30,8 & \\
\hline $35-44$ & 11 & 30,6 & 12 & 33,3 & 6 & 16,7 & 7 & 19,4 & \\
\hline$\geq 45$ & 6 & 42,8 & 4 & 28,6 & 0 & 0,00 & 4 & 28,6 & \\
\hline \multicolumn{10}{|l|}{ Fator Rh } \\
\hline Positivo & 70 & 30,7 & 55 & 24,1 & 30 & 13,2 & 73 & 32,0 & \multirow[t]{2}{*}{0,127} \\
\hline Negativo & 17 & 51,6 & 5 & 15,1 & 3 & 9,1 & 8 & 24,2 & \\
\hline \multicolumn{10}{|l|}{ Tipo de doador } \\
\hline Repetição & 68 & 43,9 & 43 & 27,7 & 14 & 9,1 & 30 & 19,3 & \multirow[t]{3}{*}{0,000} \\
\hline Esporádico & 0 & 0,00 & 0 & 0,00 & 12 & 21,8 & 43 & 78,2 & \\
\hline Foi de repetição & 19 & 37,3 & 17 & 33,3 & 7 & 13,7 & 8 & 15,7 & \\
\hline
\end{tabular}

Quanto ao tempo do último retorno dos doadores de repetição disponíveis no estudo (Gráfico 1), o eixo vertical representa o valor da função de sobrevivência, ou seja, a probabilidade de um doador de repetição não retornar para sua última doação em cada instante do tempo, medido em meses (eixo horizontal). Verificou-se que a probabilidade de não haver retornado aos seis meses é de $89,7 \%$, caindo para $76,1 \%$ quando se olha para 12 meses. Quando considerado um intervalo de dois anos, encontrou-se um grupo de aproximadamente $61,3 \%$ dos doadores que não haviam retornado.

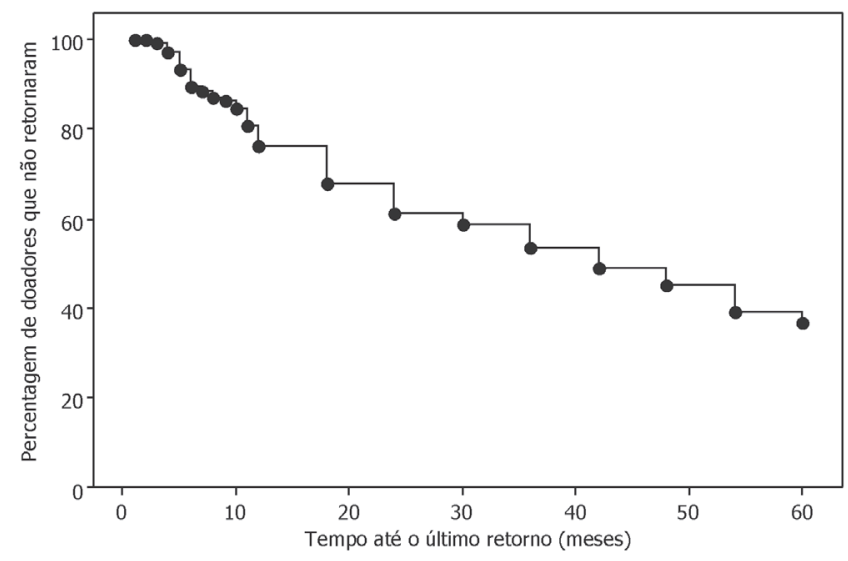

Gráfico 1. Análise de Kaplan-Meier do tempo do último retorno dos doadores de repetição disponíveis. Hemocentro Recife, Brasil, 2008

Do total de doadores pesquisados, $36,8 \%$ apresentaram uma janela de tempo de doação superior a 60 meses. O intervalo de repetição máximo encontrado foi de 120 meses.

\section{Discussão}

Encontrou-se um perfil de doador semelhante ao identificado em estudos nacionais ${ }^{(15)}$ e locais, ${ }^{(16)}$ incluindo aquele realizado, em 2007, no Hemocentro Recife ${ }^{(12)}$ : o doador voluntário, do sexo masculino, com idade entre 25 e 44 anos, segundo grau completo, que doa de forma espontânea, motivado pelo pedido de um amigo ou parente que esteja necessitando de sangue e solidariedade, merecendo destaque o significativo índice de retorno. Parcela importante referiuse à experiência de familiares que necessitaram de sangue ou possuíam familiares que já doaram sangue. A família e os amigos, como mostram vários autores, ${ }^{(3,11,15-17)}$ têm se revelado fundamentais no convencimento das pessoas para realizarem a primeira doação e se manterem doando.

$\mathrm{Na}$ descrição da história pregressa dos doadores incluídos no estudo, constatou-se o aumento da participação feminina na doação de sangue e a persistência da concentração da primeira doação no grupo populacional com idade entre 18 e 24 anos. Apesar das limitações advindas do fato de se estar lidando com doadores disponíveis em um dado momento do tempo, ${ }^{(18)} \mathrm{o}$ comportamento revelado na amostra é semelhante à tendência observada na literatura sobre o sexo e a idade à primeira doação. ${ }^{(6,8,9)}$ Certamente, avança-se no incremento do número de mulheres doadoras de sangue conforme meta governamental definida em 1998. ${ }^{(1)}$

Também foram reforçados os conhecimentos existentes acerca da relação entre a idade do doador à primeira doação e a frequência do retorno, com os mais velhos realizando maior número total de doações. ${ }^{(6,8)}$ Para Sampaio, ${ }^{(19)}$ a progressiva mudança no perfil etário da população 
brasileira fará com que os mais velhos sejam ainda mais importantes na manutenção dos estoques de sangue. Em sintonia com esta afirmação, em dezembro de 2002, a idade máxima permitida para doação aumentou, no País, de 60 para 65 anos. ${ }^{(20)}$

Confrontando outros estudos, ${ }^{(6,8,9)}$ neste o sexo não afetou de modo significativo o tempo do primeiro retorno, apesar de uma proporção maior de homens haver retornado mais precocemente. Contudo, a relação entre o padrão de comportamento de retorno e o sexo dos doadores reiterou o já observado no tocante aos homens se tornarem doadores de repetição com uma frequência significativamente maior do que as mulheres, as quais adotam um padrão de doações esporádicas. ${ }^{(6,9)} \mathrm{O}$ tipo de fator $\mathrm{Rh}$ também não se mostrou associado à primeira doação, tampouco ao comportamento de retorno. É possível que o recurso aos doadores disponíveis utilizado nesta pesquisa para analisar características do comportamento de retorno, ao mesmo tempo em que permitiu obter informações importantes sobre o mesmo, tenha obscurecido diferenças de menor magnitude.

Nos Estados Unidos, o retorno dos doadores Rh negativos é significativamente maior em comparação com aqueles Rh positivos, o que é atribuído ao forte trabalho de recrutamento do grupo. ${ }^{(6)}$ Aumentar o número de doadores $\mathrm{Rh}$ negativos, o mais raro, é um desafio a ser vencido pelos bancos de sangue, já que esse tipo é encontrado em torno $15 \%$ da população brasileira. ${ }^{(21)} \mathrm{O}$ esforço feito na tentativa de manter o estoque de bolsas de sangue mediante doação espontânea é diário, independentemente do fator Rh, incluindo a convocação direta de doadores já cadastrados para atender necessidades específicas. $\mathrm{Na}$ amostra estudada, proporção elevada do pequeno percentual de doadores convocados a comparecer ao serviço tinha o fator $\mathrm{Rh}$ negativo.

Convém chamar a atenção para a convergência com os resultados de investigações internacionais que evidenciam que o retorno precoce para a segunda doação está associado com o número total de retornos e, particularmente, com o status de doador regular. ${ }^{(6,8,9)}$ Ownby et al. ${ }^{(6)}$ ampliaram esses achados demonstrando que o tempo do próximo retorno é fortemente condicionado pelo tempo médio dos retornos prévios. Aspectos que, na opinião desses autores, indicam que os doadores deveriam ser estimulados a retornar tão precocemente quanto fosse possível para atender aos critérios de elegibilidade para ser doador de sangue. Sugestão particularmente valiosa para o Brasil, onde a exigência de uma contagem mais elevada de hemoglobina torna um desafio ainda maior converter doadores potenciais em doadores de fato e garantir o retorno de maneira regular. ${ }^{(22)}$

Para Suárez et al., ${ }^{(1)}$ os poucos estudos publicados sobre doadores regulares identificam um pequeno número de doadores de repetição. Apesar do importante percentual de retorno dos doadores, a curva de sobrevivência mostrou que podem ser grandes os intervalos de repetição, o que significa que, a cada ano, é preciso captar novos doadores para atender adequadamente, em quantidade e qualidade, a demanda crescente por sangue. Segundo pesquisa do Ministério da Saúde, em 2004, 53,5\% e 52,6\% dos doadores, respectivamente no Brasil e na região nordeste, já tinham doado cinco ou mais vezes em suas vidas, indicando um alto índice de retorno. ${ }^{(15)}$ No estudo atual, ao redor de $45,6 \%$ doaram cinco ou mais vezes. Proporção importante de doadores ou deixa de doar ou se transforma em um doador esporádico. ${ }^{(6-10)}$

Embora seja preciso empreender um esforço na captação daqueles sem antecedentes de doação, observa-se uma ênfase no desenvolvimento sistemático de estratégias que estimulem os doadores de retorno a doarem com mais regularidade. ${ }^{(6,8-11)}$ Para tanto, é preciso concentrar a atenção em subgrupos específicos porque existem diferenças nos comportamentos de doadores de primeira vez, de primeiro retorno e regulares. ${ }^{(8)}$

Schreiber et al. ${ }^{(8)}$ consideram essencial focalizar o olhar nos doadores de repetição com uma história de doações regulares, os quais seriam potencialmente mais receptivos, aumentando a efetividade do recrutamento. Afirmam que a grande maioria dos doadores de retorno realiza menos de duas doações por ano, quando é permitido um máximo de quatro doações, para o sexo masculino. Também acreditam que um aumento substancial nas doações seria alcançado com uma diminuição relativamente pequena do não retorno. Nesse sentido, esses autores ainda apontam a necessidade de que seja estudada a frequência com que doadores de primeira vez, com perfis sociodemográficos distintos, retornam para doar, além de investigados os motivos pelos quais metade dos doadores de primeira vez efetua uma única doação.

Não se deveria prescindir do conhecimento sobre os condicionantes da regularidade da doação. O hábito e a experiência prévia são considerados fatores de impacto relevante sobre o comportamento de retorno dos doadores de sangue. ${ }^{(10,16,22)}$ Para Masser et al., ${ }^{(23)} \mathrm{o}$ hábito é insuficiente para assegurar a regularidade da doação, embora seja um passo essencial na transição do recém-doador para se tornar um doador de repetição. Ainda merece ser destacada a importância da percepção dos doadores sobre as unidades de hemoterapia. Ludwig e Rodrigues ${ }^{(5)}$ argumentam que a percepção é influenciada pela confiança depositada no serviço, com a responsabilidade e competência dos profissionais, o acesso à unidade e ao atendimento, bem como a comunicação, sendo componentes centrais da satisfação de doadores.

Em um contexto onde se pretende aumentar as doações de repetição entre as mulheres, ${ }^{(1)}$ não é conveniente perder de vista as diferenças de gênero. São controvertidas as relações entre gênero e satisfação dos usuários, mas estudos constatam uma maior capacidade crítica das 
mulheres, ${ }^{(24)} \mathrm{o}$ que pode afetar as avaliações da experiência de doação. De outra perspectiva, Santos e Condeço ${ }^{(25)}$ lembram os papéis diferenciados de acordo com o gênero no que diz respeito à divisão de responsabilidades e tarefas no ambiente doméstico e as possíveis repercussões sobre a doação de sangue.

Note-se que as relações entre a aplicação de critérios de elegibilidade mais rígidos e a tendência estacionária das doações podem provocar, em curto prazo, semelhante aos Estados Unidos, ${ }^{(8,9)}$ um declínio do percentual da população de doadores. É preciso lembrar que é crônico no Brasil o déficit no número de doadores de sangue para atender à demanda de transfusões e que as estratégias adotadas na captação de doadores não produzem os resultados desejados. A mudança desse quadro também implica dedicar atenção ao monitoramento do perfil sociodemográfico e padrão de retorno dos doadores, imprescindível para identificar os grupos de doadores com maiores probabilidades de tornarem-se doadores de repetição e priorizar os aspectos que requerem ação transformadora no cotidiano do trabalho de recrutamento, atendimento e retenção de doadores.

Diante das inúmeras dificuldades encontradas pelos hemocentros em manter os estoques adequados de sangue, através de doadores de primeira vez ou de retorno, este trabalho mostra o perfil de doadores que tiveram comportamento de retorno, mostrando que é fundamental concentrar a atenção neste grupo potencialmente mais receptivo para aumentar efetividade do recrutamento.

\section{Abstract}

Objective: To analyze the profile of blood donors in respect to the period of the first donation and their return pattern to the public blood bank of Recife. Method: A retrospective study was performed of the data of 363 donors input to the Blood Bank System Database in the period from January 1, 1998 to June 13, 2008. In the analysis, the median number of donations by gender, age and schooling years were evaluated, as well as the association of the time of the first donation with the gender, age and $R h$ factor. Additionally, the return pattern of 261 of these donors (excluding 100 first time donors and two for lack of precision as to the date of return) was evaluated. Results: Approximately $77.1 \%$ were men, of whom $40.5 \%$ were from 25 to 34 years old, $47.7 \%$ had completed high school and $54 \%$ donated spontaneously. The median number of donations was higher among older men $(p=0.000)$. The participation of women increased in this period (30.6\%) compared to 1998-2000 (4.8\%), even so most first-time donors were men $(p=0.003)$. Of those who donated five or more times, $43.8 \%$ returned for the next donation at an interval of less than six months. More men than women were repeat donors $(p=0.011)$. Conclusions: the great majority of repeat donors (with a time interval between the last two donations of 13 months or less) is still not close to the maximum possible number of annual donations. It is essential to focus on this group who are potentially more receptive to increasing the effectiveness of recruitment.

Keywords: Blood donors; Blood banks

\section{Referências}

1. Souza HM, Santos LG. Programa nacional de sangue e hemoderivados - Pró-sangue. In: Bordin JO, Langhi Júnior DM, Covas DT, editores. Hemoterapia: fundamentos e prática. São Paulo: Atheneu; 2007. p.541-50.

2. Ministério da Saúde. Agência Nacional de Vigilância Sanitária. Fazendo a diferença: captando doadores voluntários de sangue. Brasília (DF): Ministério da Saúde; 2003.

3. Dorlhiac-Llacer PE. Doação de sangue e testes laboratoriais no sangue do doador. In: Chamone DAF, Novaretti MCZ, DorlhiacLlacer PE, editores. Manual de Transfusão Sangüínea. São Paulo: Roca; 2001. p.1-8

4. Gonçalez T, Sabino EC, Chamone DF. Trends in the profile of blood donors at a large blood center in the city of São Paulo, Brazil. Pan Am J Public Health. 2003;13(2-3):144-8.

5. Ludwig ST, Rodrigues ACM. Doação de sangue: uma visão de marketing. Cad Saúde Pública. 2005;21(3):932-9.

6. Ownby HE, Kong F, Watanabe K, Tu Y, Nass CC. Analysis of donor return behavior. Retrovirus epidemiology donor Study. Transfusion. 1999;39(10):1128-35.

7. Wu Y, Glynn AS, Schreiber GB, Wright DJ, Lo A, Murphy EL, et al. First-time blood donors: demographic trends. Transfusion. 2001;41(3):360-4.

8. Schreiber JB, Sanchez AM, Glynn AS, Wright DJ. Increasing blood availability by changing donation patterns. Transfusion. 2003; 43(5):591-7.

9. Schreiber GB, Sharma UK, Wright DJ, Glynn SA, Ownby HE, Tu $\mathrm{Y}$, et al. First year donation patterns predict long-term commitment for first-time donors. Vox Sang. 2005;88(2):114-21.

10. Sojka EN, Sojka P. The blood donation experience: self-reported motives and obstacles for donations blood. Vox Sang. 2008;94: 56-83.

11. Suárez IMB, Fernández-Montoya A, Fernández AR, Lópes-Berrio A, Cillero-Peñuella M. How regular blood donors explain their behavior. Transfusion. 2004;44(10):1441-6.

12. Universidade Federal de Pernambuco. Fundação de Apoio ao Desenvolvimento. Pesquisa de satisfação dos clientes da Fundação Hemope, 2007. Recife; 2007.

13. Ministério da Saúde. Agência Nacional de Vigilância Sanitária. Diretoria de Vigilância Sanitária e Ambiental. O Sistema Nacional de Informação da Produção Hemoterápica - HEMOPROD. Resolução RDC nº 149 de 14 de agosto de 2001. Republicada no Diário Oficial de 22/08/01.

14. Bustamante-Teixeira MT, Faerstein E, Latorre MR. Técnicas de análise de sobrevida. Cad Saúde Pública. 2002;18(3):574-94.

15. Ministério da Saúde. Agência Nacional de Vigilância Sanitária. Perfil do doador de sangue brasileiro. 2004/Ministério da Saúde. Agência Nacional de Vigilância Sanitária. Brasília: Editora do Ministério da Saúde; 2004.

16. Moura AS, Moreira CT, Machado CA, Neto JAV, Machado MFAS. Doador de sangue habitual e fidelizado: fatores motivacionais de adesão ao programa. RBPS. 2006;19(2):61-7.

17. Buciuniene I, Stoniene L, Blazeviciene A, Kazlauskaite R, Skudiene V. Blood donors' motivation and attitude to non-remunerated blood donation in Lithuania. BMC Public Health. 2006;6:166.

18. Fletcher RH, Fletcher SW. Epidemiologia clínica: elementos essenciais. 4a. ed. Porto Alegre: Artmed; 2006.

19. Sampaio DA. Caracterização descritiva de candidatos à doação de sangue do grupo etário de 50 a 60 anos, de ex-doadores excluídos por limite de idade, de não-doadores acima de 50 anos e análise comparativa com candidatos de 18 a 28 anos da cidade do Recife 
[tese]. São Paulo: Programa de pós-graduação em Hematologia e Hemoterapia. Escola Paulista de Medicina, Universidade Federal de São Paulo, 2001.

20. Ministério da Saúde. Agência Nacional de Vigilância Sanitária. Sancionada Lei que estabelece Política Nacional de Sangue e Hemoderivados no Brasil. 2001. [citado 2007 jun 23]. Disponível em: http:// portal.saude.gov.br/portal/arquivos/pdf/resolucao_153_2004.pdf.

21. Cozac APCNC. Sistema de Grupo Sanguineo ABO. In: Bordin JO, Langhi Jr DM e Covas DT. Hemoterapia. Fundamentos e prática. São Paulo: Atheneu; 2007. p 125-36.

22. McMahon R, Byrne M. Predicting donation among an Irish sample of donors and nondonors: extending the theory of planned behavior. Transfusion. 2008;48(2):321-31.

23. Masser BM, White KM, Hide MK, Terry DJ. The psychology of blood donation: current research and future directions. Transfus Med Rev. 2008;22(3):215-33.

24. Espiridião MA, Trad LAB. Avaliação de satisfação de usuários: considerações teórico-conceituais. Cad Saúde Pública. 2006;22(6): 1267-76.

25. Santos EC, Condeço J. Avaliação do grau de satisfação dos doadores de sangue do Hospital Distrital de Chaves. ABO-Rev Med Transf. $2002 ; 11: 5-12$.

Recebido: 20/3/2010

Aceito: 5/7/2010 